\title{
SINDROME DE GUILIAAIN-BARRÉ-STROHL
}

\author{
FATORES PROGNOSTICOS
}

\author{
ABELARDO DE QUEIROZ CAMPOS ARAUJO*- ALEXANDRA PRUFER DE QUEIROZ \\ CAMPOS ARAUJO ** - JOSE LUIZ DE SA CAVALCANTI ***
}

\begin{abstract}
RESUMO $\rightarrow$ Os autores analisaram retrospectivamente 29 paclentes internados com a sindrome de Gullain-Barré-Strohl, objetivando identificar índices de gravidade da doença no que se refere ao desenvolvimento de complicações e ao óbito. Concluíram serem indices de gravidade a presenca de sinais sensitivos, disfunção neurovegetativa, insuficiência respiratória, disfunção esfincteriana e longo tenıoo de permanência hospitalar.
\end{abstract}

Guillain-Barré-Strohl syndrome: prognostic factors.

SUMMARY - The authors analysed retrospectively 29 in-patients with Guillain-Barré-Stroh] syndrome intending to recognize severity indexes as far as the development of compications and death are concerned. Sensory signs, autonomic dysfunction, respiratory insuficiency, sphincteric disturbances and a longer time in hospital turned out to be severity indexes, when present in these patients.

Em 1916, Guillain, Barré e Strohl, ao caracterizarem como entidade mórbida a sindrome que hoje leva seus nomes, enfatizaram nāo só seus aspectos clínico-laboratoriais, descrevendo a típica alteração do líquido cefalorraquidiano (LCR) - dissociação albumino-citológica - como também chamaram a atençāo para aspectos evolıtivos, especialmente para o prognóstico favorável na grande maioria dos casos 17 . Após vários anos de experiência no trato desses pacientes, sabe-se que esta afirmativa năo é tão verdadeira, pois o indice de mortalidade da doença varia de $2 \%$ a $22 \% 1,12,13,23,25,26,35$. Reconhece-se, ainda, que 10 a $30 \%$ dos doentes requerem ventilação artificial em alguma fase da doença 10,14,33, o que contribui para aumentar 0 indice de mortalidade, decorrente de infecções devidas ao longo periodo de assistência respiratória. Assinale-se, também, a incidência de seqüelas neurológicas permanentes, em torno de $16 \% 20$.

Sendo enfermidade de curso evolutivo imprevisivel, pelo menos nas primeiras semanas 15, o estabelecimento de indices prognósticos de fácil identificação pelo clínico, reveste-se de extrema importância prática. O presente estudo foi realizado objetivando o reconhecimento de tais fatores.

\section{PACIEATTES E METODOA}

Foram analisados, retrospectivamente, os prontuários de 29 pacientes internados no HUCFF e no IPPMG, ambos da UFRJ, no periodo compreendido entre janeiro de 1978 e outubro de 1985. Selecionaram-se apenas os casos que, examinados por um ou mais neuro-

Trabalho realizado no Hospital Universitário Clementino Fraga Filho (HUCFF) e no Instituto de Puericultura e Pediatria Martagāo Gesteira (IPPMG) da Universidade Federal do Rio de Janeiro (UFRJ): * Médico do Instituto de Neurologia da UFRJ; ** Médica do IPPMG da CFrJ: *** Professor Adjunto de Neurologla da UFRJ.

Dr. Abelardo de Queiroz Campos Araújo - Praça Professor José Bernardino, 24/201 - 22621 Rio de Janeiro $R J-B r a s i l$ 
logistas, apresentaram quadro clinico-laboratorial condizente ao diagnóstico, de acordo com os critérios estabelecidos por Asbury 3. No sentido de se avaliar os principals fatores envolvidos no prognóstico da doenca, fol feito protocolo geral que recolheu dados clinicos, laboratoriais o evolutivos. A maioria desses dados, versando acerca de aspectos gerais da doença, foram suprimldos do presente estudo por terem sido objeto de monografia anteriormente apresentada 6. No estudo atual, us pacientes foram divididos em 4 grupos, a saber: 1) pacientes que obtiveram alta hospitalar; 2) pacientes falecidos durante a internaçă 3) pacientes com complicaçues durante a intermaçâ; 4) paclentes sem complicaçōes. Conceituou-se complicação como qualauer cundicão mórbida surglda no decurso da internacăo, excluídas as manifestaçōes reconhecidas como parte io quadro clínjco da sindrome de Gullain-Barré-Strohl (SGB).

Compararam-se as 4 grupos no que tange aos parámetros seguintes: u) idade, sexo e raca; b) quadro motor e sensitivo; c) presenca ou rĩo de - acometimento de nervos cranianos, sinals meningo-radiculares, distúrbios esfincterianos, distúrbios neurovegetativos; d) tempo de internaçăo. Os demais parâmetros analisados no protocolo inicial, forum excluidos degte estudo em virtude de não se mostrarem significativos para o prognóstico, em analise preliminar. Resultados das comparações entre os grupus foram analisados quanto a sus significancia estatística pelo teste da probabilidade exata de Fisher, exceção feita à média de idade e ao tempo medio de internaçá, aos quais foi aplicado o teste do $t$ de Student 32.

\section{RESULTADOS}

1) Pacientes em geral (Tabela 1) - Foram encontradas as seguintes alteracōes objetivas de sensibilidade (iniciais dos doentes entre parênteses): hipoestesia superticial e profunda com nivel sensitivo em T12 (MS); jipoestesia superficial e profunda em meia bilateralmente (JBG); hipoestesia superficial e proiunda em mels e luva bilateralmente (ISF): anestesia superficial a profunda em neia bilateralmente (LPCB); hipoestesia superficial em meia e luva bilateralmente (SMQF); hipopalestesia em membros inferiores (JMP); hipoestesia superficial em meia e luva bilateralmente (EGR). Os nervos cranianos acometidos foram: VII (12 paciestes): IX-X (11 pacjentes); III (4 pacientes); V (1 paciente); VIII (1 paciente). Alguns pacientes tiveram mais de um nervo craniano atingido, concomitantemente. Em relaçåo as disfuncỏes neurovegetativas encontramos: taquicardia sinusal (9 casos), sudorese profusa (6 casos), hipertensน̉o artelial (3 casos), bradicardia sinusal (2 casos) e hipotensão arterial (1 caso). $\mathrm{Na}$ maloria dos casos ocorreu, simultaneamente, mais de um tipo de disautonomis.

2) Comparaçăo entre o grupo de pacientes que obteve alta hospitalar e o grupo de paclentes falectdos no decurso da internacåo ('Fabela 2) - O indice de mortalidade da nossa

Parâmetros

$\begin{array}{lr}\text { Sexo masculino } & 18 \\ \text { Sexo feminino } & 11 \\ \text { Leucodermicos } & 21 \\ \text { Melanodérmicos } & 8 \\ \text { Tetraparesia } & 26 \\ \text { Paraparesia } & 3 \\ \text { Sinais sensitivos } & 7 \\ \text { Nervos cranianos } & 15 \\ \text { Sinais meningo-radiculares } & 15 \\ \text { Disfunção neurovegetativa } & 10 \\ \text { Disfunção esfincteriana } & 3 \\ \text { Insuficiência respiratória } & 5 \\ \text { Idade média (anos) } & 24 \\ \text { Tempo médio de internação (dias) } & 24 \\ \text { Número total de casos } & 29\end{array}$


amostra foi $17 \%$ (5 pucientes), tendo $83 \%$ (24 pacientes) obtido alta hospitalar. 0 b́bito ocurreu, na maiuria dos casos, em decorrência de complicaçōes infecciosas, notadamente de vias aéreas inferiores. Quando se compara o grupo dos óbitos com o grupo das altas observa-se major frequencia, estatisticamente significativa, no que tange a presenca de sinais sensitivos $(n=0,013)$, disfunçăo neurovegetativa $(p=0,004)$, insuficiência respiratória $(\mathrm{p} \rightleftharpoons 0,001)$ e maior tempo de internacão $(p<0,001)$, no primeiro grupo. Os demais parâmetros nã.o se mostraram estatisticamente relevantes $(p>0,05)$.

3) Comparacão entre o grupo de pacientes que teve algum tipo de complicacão durante - período de hospitalização, e o grupo sem complicaçóes (Tabela 3) - Complicaçoes ocorreram em 9 (31 $\%$ ) dos 29 pacientes, a saber: pneumionla (8 casos), insuficiencia respiratória aguda (5 casos), choque séptico (2 casos), coagulaçăo intravascular disseminada (2 casos), septicemia (3 casos), infeccăo urinária (2 casos), atelectasia (1 caso), tromboflebite (1 caso), hemopneumutórax ( 1 caso), sindrome da anguistia respiratória do adulto (1 caso), insuficiencla renal aguda (1 caso), úlcera ie córnea (1 caso) e escaras de decúbito (1 caso). Na maloria dos pacientes (7 no total de 9) coexistiram mais de um típo de complicacão. Confrontando-se o grupo de pacientes que teve complicaçóes com o grupo sem, nota-se, no primeiro, frequéncia maior e estatisticamente signfficativa de sinais sensitivos ( $p=0,032)$, disfunçao neurovegetativa ( $n=0,003)$, disfuncăo esfincteriana $(p=0,045)$ e maior tempo de internaça $(p<0,001)$. As diferences entre os parametros restantes não apresentaram signifícancla estatística $(p>0,05)$.

\begin{tabular}{|c|c|c|c|}
\hline Grupos / parametros & Altas & Obitos & $\mathbf{p}$ \\
\hline Sexo masculino & 14 & 4 & NS \\
\hline Sexo feminino & 10 & 1 & NS \\
\hline Leucodérmicos & 18 & 3 & NS \\
\hline Melanodérmicos & 6 & 2 & NS \\
\hline Tetraparesia & 21 & 5 & NS \\
\hline Parapkaresia & 3 & 0 & NS \\
\hline Sinats sensitivos & 3 & 4 & $=0,013$ \\
\hline Nervos cranianos & 11 & 4 & NS \\
\hline Sinais meningo-radiculares & 12 & 3 & NS \\
\hline Disfunção neurovegetativa & 5 & 5 & $=0,004$ \\
\hline Disfunção esfincteriana & 1 & 2 & NS \\
\hline Insuficiência respiratória & 1 & 4 & $=0,001$ \\
\hline Idade média (anos) & 23 & 30 & NS \\
\hline Tempo médio de internaçāo (dias) & 23 & 29 & $<0,001$ \\
\hline Número total de casog & 24 & $\mathbf{5}$ & - \\
\hline
\end{tabular}

Tabela 2 - Comparacão dos parametros segundo grupos (altas, obitos). NS, nao significativo $(\boldsymbol{p}>0,050)$.

\begin{tabular}{|c|c|c|c|}
\hline Grupos / parâmetros & Com complicação & Sem complicaçăo & $\mathbf{p}$ \\
\hline Sexo masculino & 6 & 12 & NS \\
\hline Sexo feminino & 3 & 8 & NS \\
\hline Leucodérmicus & 6 & 15 & NS \\
\hline Melanodérmicos & 3 & 5 & NS \\
\hline Tetraparesia & 9 & 17 & NS \\
\hline Paraparesia & 0 & 3 & NS \\
\hline Sinais sensitivos & 5 & 2 & $=0,032$ \\
\hline Nervos cranianos & 6 & 9 & NS \\
\hline Sinais meningo-radiculareg & 5 & 10 & NS \\
\hline Disfunçåo neurovegetativa & 7 & 3 & $=0,003$ \\
\hline Disfunçåo esfincteriana & 3 & 0 & $=0,045$ \\
\hline Idade média (anos) & 29 & 22 & NS \\
\hline Tempo médio de internaçāo (días) & 37 & 19 & $<0,001$ \\
\hline Número total de casos & 9 & 20 & 一 \\
\hline
\end{tabular}

Tabela 3 - Comparacão dos parametros segundo grupos (com e sem complicacáo). NS, não significativo $(\boldsymbol{p}>0,050)$. 


\section{COMENTARIOS}

Sabe-se, desde a década passada, que a corticoterapia não apresenta vantagens no tratamento da SGB tendo, segundo alguns autores, o inconveniente de prolongar 0 tempo de permanência hospitalar e predispor a formas recorrentes da doença $\mathbf{1 9 , 2 3}$. Isto fez com que se buscassem outras abordagens terapêuticas que pudessem se mostrar eficazes. O papel teórico da plasmaférese na SGB surgiu, principalmente a partir da demonstração, por Cook \& Dawling 8 , no soro desses pacientes, da existência de fatores humorais mielinotóxicos. Este fato fez surgir na literatura inúmeros trabalhos, em sua maioria não controlados, versando a eficácia de tal método. Finalmente, em 1985, - Guillain-Barré Study Group, formado por diversos centros neurológicos dos EUA, realizou investigação prospectiva randomizada em 245 pacientes gravemente acometidos pela doença. O trabalho concluiu pela eficácia da plasmaférese, especialmente se utilizada em pacientes graves, com menos de 7 dias de doença e dependentes de repirador 33 Tal conduta passou a ser recomendada, desde então, como o procedimento terapêutico mais eficaz 16 .

Uma vez que a maioria dos pacientes se recupera espontaneamente sem qualquer sequiela, é óbvia a necessidade de se estabelecerem critérios de gravidade, capazes de selecionar os enfermos com pior prognóstico, que se beneficiariam da plasmaférese. Tai fato assume uma importância ainda maior em nosso meio pois, esta forma de tratamento, além de envolver desconforto e riscos para o doente, necessita de maquinaria especial, suporte clínico intensivo, pessoal especializado e, conseqüentemente, custos financeiros elevados. Poucos são os trabalhos na literatura que se propōem a responder a essas questóes de modo especifico. Embora alguns artigos tenham tentado correlacionar certos aspectos clínicos com o prognóstico, nenhum foi capaz de identificar de maneira definitiva o grupo de pior prognóstico nos estágios iniciais da doença. Segundo Osler \& Sidell 27, seriam fatores de bom prognóstico a presença de neuropatia exclusivamente motora e a ausência de distúrbios esfincterianos. Tal afirmativa foi contestada por Marshall 24, o qual examinou 35 pacientes, alguns dos quais com grave distúrbio sensitivo e disfunção esfincteriana. De acordo com outros autores 23,29 . a presença de grave déficit motor, traria mau prognóstico quanto à ocorrência de sequielas. Trabalhos com populaçōes pediátricas 11,29 concluíram ser de valor prognóstico o tempo decorrido para o início da fase de recuperaçāo. De acordo com Eberle et al.11, em avaliação retrospectiva de 47 crianças, intervalo de tempo superior a 18 dias, desde o déficit máximo e o início da recuperação, estaria associado a maior incidência de sequielas. Segundo outros autores, correlacionar-se-iam com pior prognóstico a presença de amiotrofia 20,23 , ou de fibrilações profusas à eletroneuromiografia, indicativas de degeneração axonal 26 .

Não foi possivel incluir, entre os parâmetros analisados no presente trabalho, dados eletroneuromiográficos, dada à inconstância desses exames nos prontuários consultados. Winer et al.35, avaliando retrospectivamente 71 pacientes com SGB, os quals haviam sido estudados em dois trabalhos anteriores $\mathbf{1 5 , 1 9}$, concluíram que nos estágios iniciais da doença seriam fatores de mau prognóstico a presença de grave déficit motor e a necessidade de ventilação artificial. Seu trabalho não é isento de críticas, principalmente quando se leva em conta a inclusão apenas de pacientes seriamente acometidos pela enfermidade. Trabalho interessante foi realizado por Gruener et al.16: estudaram 24 pacientes submetidos à plasmaférese, intencionando elucidar as características peculiares do grupo mais beneficiado pelo método. Concluiram serem indices predizentes de boa resposta a idade mais jovem e a ausência de evidência de bloqueio de condução nervosa distal à eletroneuromiografia.

A tabela 1 permite o conhecimento do perfil geral dos pacientes estudados no presente trabalho: o fato do sexo masculino ser mais acometido é concordante com outros autores 12,13,22; em relação à média de idade, o grupo apresentou faixa etária um pouco abaixo da descrita na literatura $5,7,31$. A explicação para este dado está em que uma das instituições envolvidas é centro de referência para atendimento diferenciado de casos pediátricos (IPPMG-UFRJ), acarretando, com isto, maior peso de representatividade na faixa infantil. Na maioria dos pacientes o quadro motor caracterizou-se por tetraparesia simétrica, sem predomínio proximal ou distal. Não foram observadas amiotrofias, o que pode ser atribuido à rapidez na progressão dos sintomas. A hipo ou arreflexia profunda esteve presente como sinal marcante, em todos os pacientes, nos 4 membros, mesmo nos não paréticos. Tal fato já é clássico, sendo descrito em livros-texto da especialidade 1 . Apenas $7(24 \%)$ pacientes apresentaram distúrbios sensitivos objetivos. $\mathrm{Na}$ maioria dos doentes, no entanto, o acometimento objetivo esteve ausente ou foi menos pronunciado que o subjetivo, o que está 
de acordo com a literatura 21. Sabe-se que tais alterações, quando presentes, envolvem principalmente a sensibilidade profunda. Alterações da sensibilidade superficial implicam em destruição axonal e estão presentes em casos de evolução mais prolongada 21. Isto explica a inclusão dos fenômenos sensitivos objetivos como um dos índices de mau prognóstico, tanto no que diz respeito ao óbito quanto ao desenvolvimento de complicações (Tabelas 2 e 3 ).

Dos 29 pacientes, $15(52 \%)$ tiveram um ou mais nervos cranianos acometidos. Outros autores referem percentagens que variam de $31 \%$ a $95 \%$, máxima quando se selecionam apenas os casos mais graves $2,4,5,7,13,18,26,30,31,34$. Dos nervos acometidos, os mais freqüentes foram o VII, seguido do IX-X. $\mathrm{Na}$ literatura, são díspares as percentagens quanto ao acometimento de cada nervo craniano, havendo, entretanto, concordância no que se refere ao maior comprometimento do VII $2,4,5,7,13,18,25,30,31,34$. Não houve, na amostra estudada, relevância estatística no que se refere a inclusão de acometimento de nervos cranianos dentre os fatores de mau prognóstico, tanto para o desenvolvimento de complicações, quanto para a evolução para o óbito. Isto vai de encontro, parcialmente, à afirmativa de Palacios 28 , o qual, estudando 339 pacientes com SGB, referiu como indices de mau prognóstico o acometimento do $\mathrm{X}$ nervo, além da rapidez na instalação da sintomatologia e o comprometimento respiratório. Os dados obtidos na presente casuística são semelhantes aos de Winer et al.35. Sinais meningo-radiculares (rigidez de nuca e sinais de Lasègue, Kernig e Brudzinski) foram observados em $15(52 \%)$ pacientes. A presença de tais sinais evidencia o comprometimento inflamatório das raizes dos nervos não se relacionando, nos doentes estudados, com a celularidade do LCR ou com o prognóstico final. Distúrbios esfincterianos ocorreram, transitoriamente, em $3(10 \%)$ dos pacientes. Tal acometimento é considerado incomum pela maioria dos autores, estando presente em percentagens que variam de $2 \%$ a $31 \% 18,22,25$. Encontrou-se correlação, estatisticamente significativa, entre a presença desses distúrbios e o desenvolvimento de complicações no decurso da internação, sem associação de maior incidência de óbitos. Identificaram-se sinais de acometimento neurovegetativo em $10(34 \%)$ pacientes, sendo os mais freqüentes a taquicardia sinusal e a sudorese profusa. E bem conhecida a presença de disfunção autonômica em pacientes com SGB 1,21. Taquicardia sinusal, algumas vezes persistente, ocorre em até $50 \%$ dos casos mais graves. Juntamente com a hipertensão arterial, tem sido interpretada como decorrente da hiperatividade simpática, evidenciada pelo aumento da excreção urinária de catecolaminas 9. Tal explicação é perfeitamente plausivel também no que se refere à sudorese profusa. Houve nítida associação, na amostra estudada, entre a presença de sintomas neurovegetativos e o prognóstico, tendo sido, a disautonomia, mais freqüente em pacientes que evoluiram para o óbito ou que apresentaram complicações (Tabelas 2 e 3 ).

Sabe-se que todo o paciente com SGB é candidato ao desenvolvimento de insuficiência respiratória aguda. Destes, 10 a $30 \%$ requerem assistência ventilatória mecânica 10,14,33. Estudo retrospectivo da Clínica Mayo 14 revelou frequêencia de $17 \%$ de insuficiência respiratória nesses casos. Já no Massachussetts General Hospital, 30\% dos doentes necessitaram de ventilação artificial, por tempo médio de 49 dias 33 . Insuficiência respiratória ocorreu em $5(17 \%)$ dos pacientes estudados, dos quais $4(80 \%)$ faleceram. Comparando-se os grupos Óbitos $\times$ Altas (Tabela 2), nota-se diferença, estatisticamente significativa, no que tange à presença de insuficiência respiratória no primeiro grupo. Assim sendo, a insuficiência respiratória aguda é outro fator de mau prognóstico, provavelmente pelas complicações advindas da entubação e/ou traqueostomia prolongadas e pelo curso protraído da falência ventilatória na SGB 14. Pelo fato de já ter sido incluida no grupo das complicaçōes em geral, a insuficiência respiratória não foi considerada como parâmetro isolado na tabela comparativa de complicações (Tabela 3). No Massachussetts General Hospital a média de tempo de internação hospitalar nos casos de $\mathrm{SGB}$, foi 61 dias 33 . O tempo médio de internação destes 29 pacientes situou-se em torno de 24 dias. No grupo dos óbitos, este período atingiu 29 dias e no grupo das complicações, 37 dias. As diferenças observadas foram estatisticamente significativas, isto é, maior tempo de internação se relacionou com maior índice de complicações e óbitos (Tabelas 2 e 3).

Em resumo, após análise retrospectiva de 29 pacientes com SGB, não selecionados quanto à gravidade, foram fatores de mau prognóstico quanto ao risco de óbito e desenvolvimento de complicações a presença de sinais sensitivos, disfunção neurovegetativa, insuficiência respiratória, maior tempo de internação e disfunção esfincteriana (esta última apenas no que tange a propensão ao desenvolvimento de complicações, não sendo significativamente mais freqüente no grupo que evoluiu para óbito). Apesar dos cuidados que os Autores tiveram para evitar homogeneização do 
modelo, os dados obtidos devem ser considerados dentro do contexto em que foram produzidos, isto é, não devem ser generalizados, pois a amostra é relativamente pequena e obtida em ambiente hospitalar de referência para casos graves. Tal fato não invalida o estudo em si, pois o que se buscou foram indices de gravidade da doença dentro de tal amostra. Acredita-se, portanto, que ao menos no que se refere à populaçào estudada, tais fatores de mau prognóstico indicam a necessidade de conduta mais agressiva e precoce, tornando estes pacientes candidatos à plasmaférese, ressalvando-se as recomendaçốes do Guillain-Barré Study Group 33.

\section{REFERENCIAS}

1. Adams RD, Victor M - Principles of Neurology. Ed 3. McGraw-Hill, New York, 1985.

2. Anderson $\mathbf{T}$, Siden $\mathbf{A}-\mathbf{A}$ clinical study of the Guillain-Barré syndrome. Acta Neurol Scand 66:316, 1982.

3. Asbury AK - Diagnostic considerations in Guillain-Barré syndrome. Ann Neurol 9 (suppl) :1, 1981 .

4. Bak $\mathbf{P}-$ Guillajn-Barrê syndrome in a Danish county. Neurology 35:207, 1985.

5. Beghi E, Kurland L, Mulder DW, wiederholt W - Guillain-Barré syndrome: clinicoepidemiologic features and effect of influenzae vaccine. Arch Neurol 42:1053, 1985.

6. Cavalcanti JLS, Arauio AQC, Araujo APQC - Sindrome de Guillain-Barré-Strohl: análise de 29 casos. Monorrafia. Academia Nacional de Medicina, Rio de Janeiro, 1987.

7. Codeceira AJr, Ferreira MLB, Marques PRB, Mesquita SD, Vilela MLG, Valença Mos, Ataide L - Polirradiculoneurites inflamatorias. Neurobiologia (Recife) 48:133, 1985.

8. Cook SD, Dawling PC - The role of autoantbody and immune complexes in the pathogenesis of Guillain-Barré syndrome. Ann Neurol 9(suppl):70, $19 \delta 1$.

9. Davies AG, Dingle HR - Observations on cardinvascular and neuroendocrine disturbance in the Guillain-Barré syndrome. J Neurol Neurosurg Psychiat 35:176, 1972.

10. Dowling PC, Memonna JP, Cook SD - Guillain-Barré syndrome in greater New YorkNew Jersey. J Am Med Assoc 238:31\%, 1977.

11. Eberle E, Brink J, Azen J, White D - Early predictors of incomplete recovery in children with Guillain-Barré polyneuritis. J Pediatr 86:356, 1975.

12. Eiben RM, Gersony WM - Recognition, prognosis and treatment of the Guillain-Barré syndrome (acute idiopatic polyneuritis). Med Clin North Am 47:1371, 1963.

13. F'reitas MRG. Nevares MT - Sindrome de Guillain-Barré: estudo clínico de pacientes internados no Hospital Universitário Antonio Pedro de 1972 a 1986. Rev Bras Neurol 23:75, 1987.

14. Gracey DR, MCMichan JC, Divertie NB, Howard FM - Respiratory failure in Guillain-Barré syndrome: a 6 year experience. Mayo Clin Proc 57:742, 1982.

15. Greenwood RJ, Newbom-Davis J, Hughes RAC, Aslan S, Bowden AN - Controlled trial of plasma exchange in acute inflammatory polyradiculoneuropathy. Lancet 1:877, 1984.

16. Gruener G, Bosch EP, Strauss RG, Klugman M, Kimura J - Prediction of early beneficial response to plasma exchange in Gullain-Barré syndrome. Arch Neurol 44:295, 1987.

17. Guillain G, Barré JA, Strohl A - Sur un syndrome de radiculonevrite avec hyperalbuminose du liquide céphalo-rachidien sans reaction cellulaire: remarques sur les caractères cliniques et graphiques des reflexes tendineux. Bull Mem Soc Med Hop Parls 40:1462, 1916.

18. Haymaker W, Kernohan JW - The Landry-Guillain-Barre syndrome: a clinicopathologic report of 50 fatal cases and a critique of the literature. Medicine (Baltimore) 28:59, 1949.

19. Hughes RAC, Newsom-Davis J, Perkin GD, Fierce JM - Controlled trial of prednisolone in acute polyneuropathy, Lancet $2: 750,1978$. 
20. Hughes RAC, Winer JB - Guillain-Barré syndrome. In Mathews WB, Glaser GH (eds): Recent Advances in Clinical Neurology, 4. Churchill Livfngstone, London, 1984.

21. Koski CL - Guillain-Barré syndrome. Neurologic Clinics 2:235, 1984.

22. Levy JA, Sanvito W - Polirradiculoneurites: considerações sobre 111 casos. Rev Hosp Clin Fac Med S Paulo 20:239, 1965.

23. Loffel NB, Rossi LN, Mumenthaler M, Lutschg J, Ludin HF - The Landry-Guillain-Barré syndrome: complications, prognosis and natural history in 123 cases. $J$ Neurol Scl $33: 71,1977$.

24. Marshall J - The Landry-Guillain-Barré syndrome. Brain 86:56, 1963.

25. McFurland RH, Heller GL - Guillain-Barré disease complex: a statement of diagnostic criteria and analysis of 100 cases. Arch Neurol 14:196, 1966.

2t. Mcleod JG - Electrophysiologrical studies in Guillain-Barrê syndrome. Ann Neurol 9 (suppl) :20, 1981.

27. Osler ID, Sidell AD - The Gilillain-Barré syndrome: the need for exact diagnostic criterla. N Engl J Med 262:964, 1960.

28. Palaclos E - Sindrome de Guillain-Barré: estudio clínico de 339 pacientes. Acta Med Colomb $7: 69,1982$.

29. Pleasure DE, Lovelace RE, Duvoisin RC - The prognosis of acute polyradiculoneuritis. Neurology 18:1143, 1968.

30. Ravn $\mathbf{H}$ - The Landry-Guillain-Barré syndrome: a survey and a clínical report of 127 cases. Acta Neurol Scand 43(suppl 30):1, 1967.

31. Soffer D, Feldman S, Alter M - Epldemiology of Gullain-Barré syndrome. Neurology $28: 686,1978$.

32. Sokal RR, Rohlf FJ - Introduction to Blostatistics. Ed 2. Freeman, San Francisco, 1973.

33. The Gułllain-Burré Syndrome Study Group - Plasmapheresis and acute Guillain-Barré syndrome. Neurology $35: 1096,1985$.

34. Tosta ED, Brasil JP, Figuelredo MAA - $O$ uso de corticosteróldes na sindrome de Gutllain-Barrê: estudo de 51 casos. Ara Neuro-Psiquiat (São Paulo) 44:117, 1986.

35. Winer JB, Hughes RAC, Greenwood RJ, Perkin GD, Healy MrJ - Prognosis in Guillain-Barré syndrome. Lancet 2:1202, 1985. 